\title{
Direct Transformation of Edible Vegetable Waste into Bioplastics
}

\author{
Ilker S. Bayer, ${ }^{\dagger} *$ Susana Guzman-Puyol, ${ }^{\dagger}$ José Alejandro Heredia-Guerrero, ${ }^{\dagger}$ Luca Ceseracciu, ${ }^{\dagger}$ \\ Francesca Pignatelli, ${ }^{\dagger}$ Roberta Ruffilli, ${ }^{\ddagger}$ Roberto Cingolani, ${ }^{\S}$ and Athanassia Athanassiou ${ }^{\dagger, *}$ \\ ${ }^{\dagger}$ Smart Materials, Nanophysics, Istituto Italiano di Tecnologia, Via Morego, 30, Genova, 16163 Italy \\ ${ }^{\ddagger}$ Nanochemistry, Istituto Italiano di Tecnologia, Via Morego, 30, Genova, 16163 Italy \\ ${ }^{\S}$ Istituto Italiano di Tecnologia, Via Morego, 30, Genova, 16163 Italy
}

Supporting Information

ABSTRACT: Bioplastics with a wide range of mechanical properties were directly obtained from industrially processed edible vegetable and cereal wastes. As model systems, we present bioplastics synthesized from wastes of parsley and spinach stems, rice hulls, and cocoa pod husks by digesting in trifluoroacetic acid (TFA), casting, and evaporation. In this way, amorphous cellulose-based plastics are formed. Moreover, many other natural elements present in these plants are carried over into the bioplastics rendering them with many exceptional thermo-physical properties. Here, we show that, due to their broad compatibility with cellulose, amorphous cellulose can be naturally plasticized with these bioplastics by simply mixing during processing. Comparison of their mechanical properties with that of various petroleum based synthetic polymers indicates that these bioplastics have equivalent mechanical properties to the nondegrading ones. This opens up possibilities for replacing some of the nondegrading polymers with the present bioplastics obtained from agro-waste.

\section{INTRODUCTION}

Plastics are probably the most frequent materials that inhabitants of developed countries come across every day. This sounds reasonable if we consider the endless list of plastic objects that surround us, like bags, beverage bottles, packages of food, elements and packaging of electronic appliances, parts of automobiles, sections of buildings, furniture, clothing elements, etc. According to the association of plastics manufacturers, Plastics Europe, the production of plastics reached 288 million tons in 2012 worldwide, which is even more impressive if we compare it with their production 10 and 20 years ago, that was just beyond 200 and 100 million tons, respectively. The astonishing growth of plastics usage is totally justified by their remarkable physical properties, easy processing, and low cost. Unfortunately, the drawbacks of this explosive surge in plastics production are progressively becoming more and more noticeable, competing with the respective advantages. The first and possibly most obvious negative impact is the huge amount of waste generation. The US Environmental Protection Agency reports that the United States in 2011 only generated 32 million tons of plastic waste, representing $12.7 \%$ of the total municipal waste. Note that plastic wastes compared to the rest do not biodegrade but persist for hundreds or even thousands of years after being thrown away. Not only do they not biodegrade but also they break down over time due to environmental conditions (UV light, heat, etc.) into toxic microfragments leaching easily into the surrounding ecosystems. ${ }^{1}$ For instance, plastic fragments can contain harmful
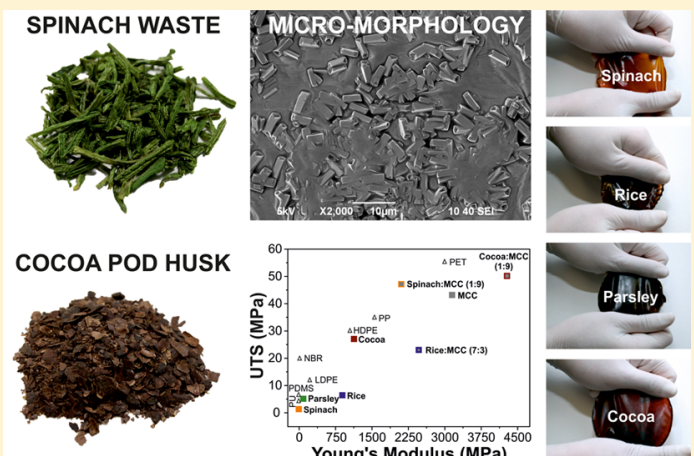

Papristey

cocoa

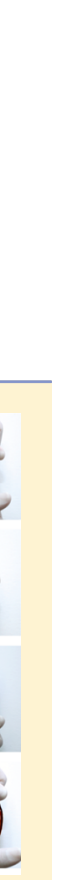


generated per annum. ${ }^{11}$ This biomass, being rich in cellulose, has been considered as a potential feedstock for the production of biofuels $^{12}$ with a main drawback that the contained cellulose is not instantly available for enzymatic digestion due to biomass recalcitrance. ${ }^{13,14}$ Cellulose is the most abundant renewable polymer in nature, being the main building component of our planet's vegetation. It is a crystalline unbranched polymer with straight chain conformation, ideal for the formation of strong fibers. Making pure cellulose bioplastics from biosources is still highly challenging due to the fact that it has a strong and highly structured intermolecular hydrogen bonding network that cannot be melt or dissolved by standard processes such as thermoforming or dissolution, ${ }^{14-17}$ and for this reason it is principally used in industrial applications in the form of cellulose derivatives, such as cellulose esters or ethers. To regenerate cellulose from its derivatives usually requires extra time and costly chemical purification steps. ${ }^{18} \mathrm{~A}$ verified but overlooked way to process cellulose is through interaction with TFA, ${ }^{17,19}$ but no one has attempted so far to obtain biopolymers from lignocellulosic biomass through TFA reactions. TFA is a naturally occurring organic acid (over 200000000 tons present in the oceans ${ }^{20,21}$ ) of low toxicity and is reported to be biodegraded by microbial action. ${ }^{21}$ Moreover, it is volatile (recyclable by distillation) and is miscible with many organic solvents as well as water, a rare quality considering other mineral acids. Even though TFA's highly oxidizing nature, strong acidity, and high volatility cause safety concerns in large scale use, a properly designed closed loop process can be used to reclaim TFA vapors by condensation or distillation. TFA is also a powerful solvent for various biomacromolecules such as proteins. ${ }^{22}$ Here we use TFA as a unique solvent for edible vegetable residues that can cosolubilize cellulose with other contained organic matter. With this method, cellulose-based bioplastics from wastes of various edible vegetables and cereals with diverse bio-origins are prepared without the need of cellulose regeneration. The new biopolymers exhibit broadly tunable thermo-mechanical properties depending on the biosource but also make cellulose potentially more accessible to the enzymes by overcoming biomass recalcitrance. ${ }^{23}$ The mechanical properties of the produced bioplastics are comparable to many synthetic polymers, in view of substituting part of the latter with degradable bioplastics having matching properties.

\section{EXPERIMENTAL SECTION}

Materials. Reagent grade trifluoroactic acid (TFA), microcrystalline cellulose (MCC), and cellulose acetate were purchased from Sigma-Aldrich and used as received. Parsley and spinach stems, cocoa pod husks and rice hulls were provided by local vegetable processors as unusable waste from their production line. Deionized water was obtained from a Milli-Q Advantage A10 Ultrapure Water Purification device.

Fabrication and Film Casting Process. MCC, parsley and spinach stems, cocoa pod husks and rice hulls were dried in an oven at $40{ }^{\circ} \mathrm{C}$ overnight in order to eliminate excess adsorbed water even though they were received from the respective producers in dehydrated format. Note that parsley and spinach stems were washed extensively in the plant of the processor. This process not only cleans the vegetables but also removes residual sugars and alcohols that might be present in the plant cell walls. Each material was dispersed in TFA at room temperature. Solutions of $3 \%$ by weight of solids in TFA were prepared in $60 \mathrm{~mL}$ glass vials using a special chemical hood designed for handling acids. Vials were sealed with Parafilm and were placed in a benchtop lab shaker for a number of days. Depending on the plant, the vegetable wastes started to dissolve after 3 days. In order to minimize the undissolved portion up to the plant wasted could be left in solution up to 2 weeks. MCC however, formed a clear viscous solution in TFA after 3 days. Similarly, parsley stem wastes also were found to form a viscous solution after 3 days. Next, the obtained solutions were centrifuged in order to remove any residuals and the remaining clear solutions were cast in plastic Petri dishes for making free-standing films. The solutions were also spin coated on microscope glass slides as coatings. In order to standardize the Bioplastic forming process and to have homogeneous solutions, all the films were cast after 29 days of aging of the biowaste powders in TFA. After casting, the films were kept in a chemical hood until all the solvent evaporated. This step was followed by keeping the films under $60 \%$ relative humidity for 2 days in order to remove any residual TFA from the films.

Chemical and Morphological Characterization. The chemical constitution of the amorphous cellulose and vegetable waste films were characterized by the Fourier transform infrared attenuated total reflectance spectroscopy (FTIR-ATR). Spectra were recorded at room temperature using a Nicolet 400D Impacts spectrometer (FTIR Brucker V70) in the range of $4000-600 \mathrm{~cm}^{-1}$ with a resolution of 4 $\mathrm{cm}^{-1}$. A total of 64 scans were signal-averaged to reduce spectral noise. The spectra were particularly useful in understanding whether or not microcrystalline cellulose or vegetable waste films were acetylated via interactions with TFA. For each film, a minimum of three measurements from different samples were conducted in order to ascertain the repeatability of the spectral results. The crystallinity of the films was analyzed by X-ray diffraction spectroscopy using Rigaku SmartLab X-ray diffractometer equipped with a copper rotating anode. Similarly, measurements were repeated on different samples obtained from the same vegetable waste in order to confirm repeatability. The $\mathrm{X}$-ray source was operated at $40 \mathrm{kV}$ and $150 \mathrm{~mA}$. A Gobel mirror was used to obtain a parallel beam and to suppress $\mathrm{Cu} \mathrm{K} \alpha$ radiation (1.392 $\AA$ ). The measurements were performed using a $2 \theta$ scan. Scanning electron microscope (SEM) images were acquired detecting back scattered electrons on a JEOL JSM-6490LA, working at a pressure of $40 \mathrm{~Pa}$ to reduce sample charging. Energy dispersive X-ray analysis (EDX) was also conducted using during SEM imaging in order to investigate the presence of other elements in the bioplastics originating from the vegetable waste. Atomic force microscope measurements were obtained by a Park System AFM instrument (XE-100) in true noncontact mode. The images were acquired in air using an antivibration table (Table Stable TS-150) and an acoustic enclosure. Single-beam silicon cantilevers tips (PPP-NCHR-10) were used for the data acquisition with less than $10 \mathrm{~nm}$ nominal radius and $42 \mathrm{~N} / \mathrm{m}$ elastic force constant for high sensitivity. The resonance frequency was defined around $280 \mathrm{kHz}$. The scan rate was between 0.2 and $1.0 \mathrm{~Hz}$ depending on the sample roughness.

Thermo-Mechanical Characterization. The decomposition of the Bioplastic samples was investigated by means of thermogravimetric analysis using a Mettler Toledo thermogravimetic/differential scanning calorimetry (TGA/DSC). Measurements were performed on 3-5 mg Bioplastic samples in an aluminum pan at a heating rate of $10^{\circ} \mathrm{C} / \mathrm{min}$, from 30 to $600{ }^{\circ} \mathrm{C}$ in nitrogen atmosphere in order to determine the thermal stability of the films. The weight loss (TG curve) and its first derivative (DTG curve) were recorded simultaneously as a function of time and temperature. Mechanical properties of the bioplastics were measured with an Instron dual column tabletop universal testing System 3365 having $5 \mathrm{kN}$ capacity, $1000 \mathrm{~mm} / \mathrm{min}$ maximum speed and $1193 \mathrm{~mm}$ vertical test space. The tensile measurements were conducted according to ASTM D 882 Standard Test Methods for Tensile Properties of Thin Plastic Sheeting. Up to seven different biopolymer film samples from each vegetable waste were measured and measurements were averaged in order to calculate Young's modulus and elongation at break under ambient conditions.

Water Adsorption Measurements. Water uptake measurements on amorphous cellulose and vegetable wastes bioplastics were also carried out in order to compare their behavior with commercial cellophane film and cellulose acetate film cast from acetone solutions in our laboratory. Dry samples were weighed on a sensitive electronic balance and then they were placed in different humidity chambers. Samples were dried by conditioning in a desiccator for several days. 


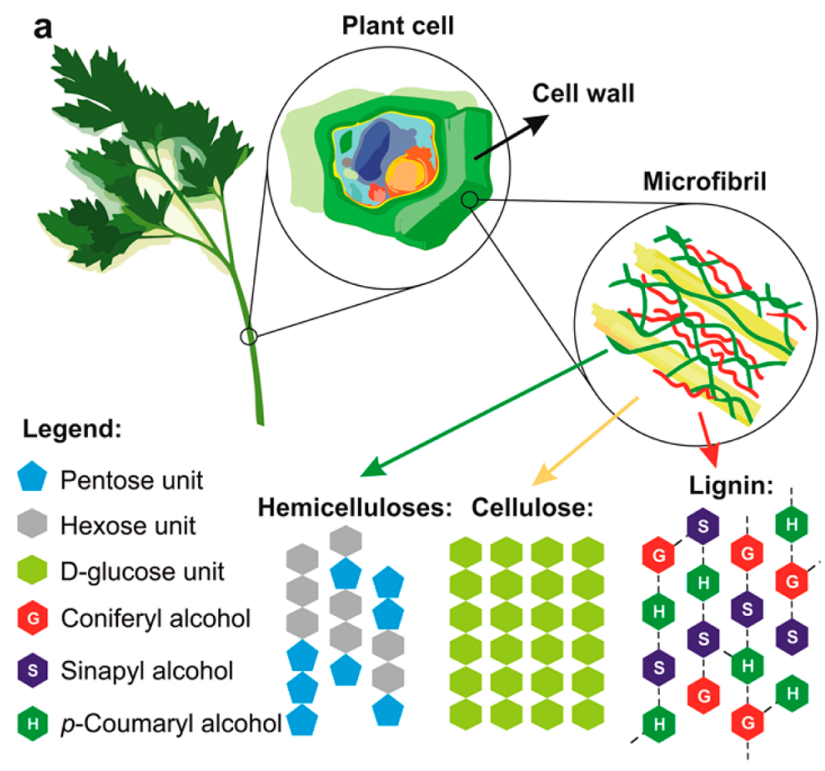

b Waste
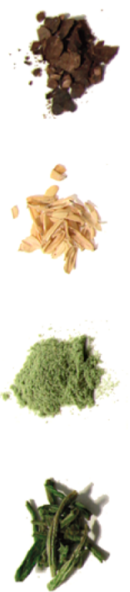

Biocoating
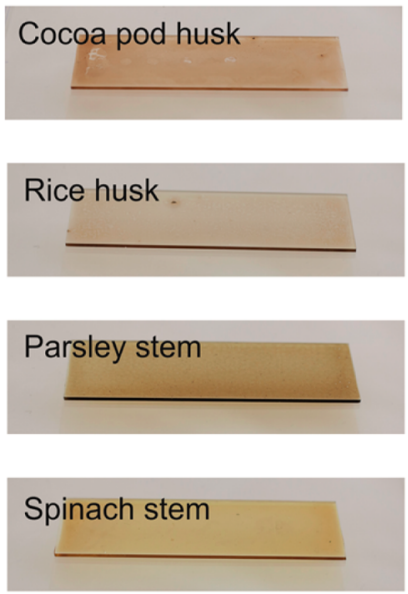
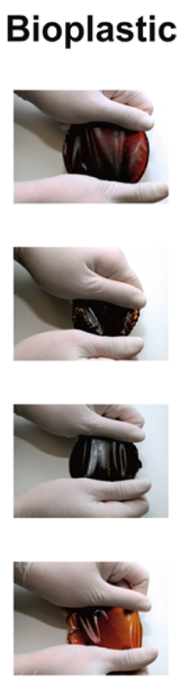

C
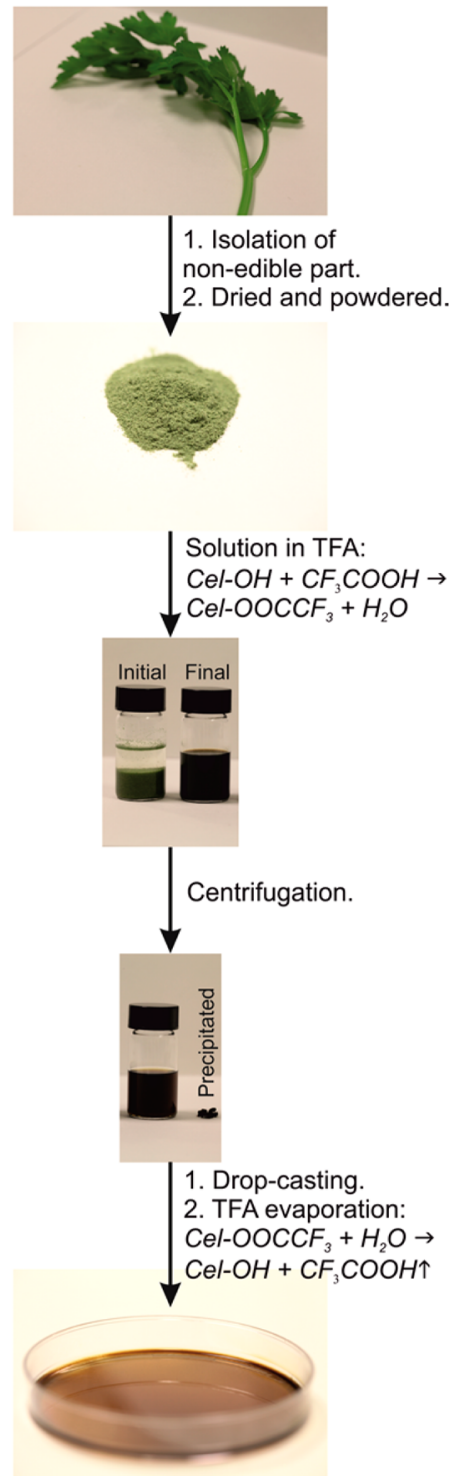

Figure 1. Edible plant waste products. (a) Schematic of the main components (hemicellulose, cellulose and lignin) of inedible plant wastes and their distribution at different scales. (b) Different edible plant wastes in diverse forms used in this study. Also, resultant biocoating and Bioplastic products are shown. (c) Bioplastic production process from plant wastes to final films. Pulverized vegetable waste is dissolved in TFA after a proper aging time is reached the solution is cast into a Petri dish. Centrifugation can be used in order to eliminate undissolved parts.

The following humidity conditions were set: $0 \%, 15 \%, 45 \%, 85 \%$, and $100 \%$. After remaining in humidity chambers for several days each film was weighed and the amount of adsorbed water was calculated based on the initial dry weight as the difference.

\section{RESULTS AND DISCUSSION}

The parsley and spinach stems, the husks from cocoa and the hulls of rice used in this work are typical wastes of edible plants and were obtained from local industrial producers. They contain considerable amount of cellulose, hemicellulose, and lignin that although variable by bio-origin, on average account for $40 \%, 25 \%$, and $20 \%$, respectively, of the total weight ${ }^{24-26}$ (Figure 1a). In addition, these processed plant wastes contain considerable amount of antioxidants, essential oils, vitamins, minerals and flavonoids (pigments) depending on their origin. ${ }^{27}$ Parsley and spinach stem wastes were pulverized and chopped, respectively, by the manufacturer during processing, whereas rice hulls and cocoa pod husks were used without further mechanical processing (Figure 1b). As shown in Figure $1 \mathrm{c}$ the processed wastes were mixed into anhydrous TFA (3 wt $\%)$ and left for a specific number of days. Each processed plant residue requires different time in TFA to form a solution suitable for solvent-casting. For instance, parsley stem powder can form a film from solution after 3 days in TFA, whereas spinach stems require up to 2 weeks in TFA. This can be mainly attributed to the physiology of the plant residue, moisture content and accessibility of bound cellulose by TFA. In plants, in addition to lignin and hemicellulose, pectin and waxes are also present with cellulose in the cell walls. ${ }^{27}$ For instance, in cocoa pod husk various types of fatty acids (cocoa butter) and substantial amount of pectin are also present that can slow down interaction with TFA. ${ }^{28}$ These solutions were used to make both coatings and free-standing films as shown in Figure $1 \mathrm{~b}$. Note that the nondissolvable portion isolated from centrifuging process, shown in Figure 1c, can still be valorized by redispersing in TFA. Such an example for spinach waste is 
shown in Figure S1 (Supporting Information). Such residual films could be suitable as feedstock for enzymatic plant waste processing for instance.

Amorphous Cellulose Plastics from Microcrystalline Cellulose. For a thorough comprehension of the TFAcellulose interaction mechanism, we performed experiments also with pure microcrystalline cellulose. We found that under ambient conditions amorphous cellulose bioplastics can be cast from TFA solutions after 3 days of aging. Analysis using Fourier transform infrared (FTIR) spectroscopy indicated that dried films are trifluoroacetylated immediately after their formation and evaporation of TFA, with the presence of a significant peak at $1790 \mathrm{~cm}^{-1}$ in the spectrum. ${ }^{29}$ However, this esterification was found to be only temporary as it was spontaneously removed in the presence of water or in humid atmosphere $(60 \% \mathrm{RH})$ after $1-2$ days as shown in Figure 2 . As seen in

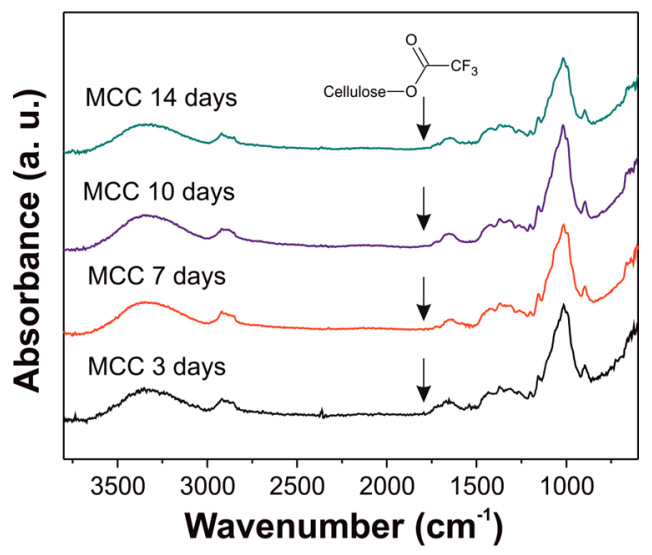

Figure 2. FTIR spectra of pure cellulose bioplastics cast from solutions in TFA at different aging days $(3,7,10$, and 14 days). No trifluoroacetylated residue bands (at $1790 \mathrm{~cm}^{-1}$ ) appear after keeping the films under ambient conditions for few days.

Figure 2, the FTIR spectra of pure cellulose bioplastics from solutions in TFA at different aging days $(3,7,10$, and 14 days) show no significant spectral differences when compared. Typical spectral features of cellulose are observed with no trifluoroacetylated residue bands corresponding to $1790 \mathrm{~cm}^{-1}$ after keeping the films under ambient conditions for few days. Dried films could not be dissolved in common solvents like other cellulose derivatives, such as cellulose acetate, confirming the nonpermanent functionalization.

Detailed atomic force microscope (AFM) measurements conducted on the transparent Bioplastic films obtained from pure microcrystalline cellulose, as a function of different aging time in TFA, indicated amorphous film surface morphology with no formation of crystalline cellulose domains or nanowhiskers as shown in Figure 3. A number of AFM images for each cellulose sample corresponding to the different aging times in TFA were collected in order to analyze the surface roughness. Films were cast on smooth silicon wafers for AFM measurements. For the 3-day aged samples (Figure 3a) an average surface roughness of $1.6 \mathrm{~nm}$ was recorded. As the aging days increased (Figure $3 b$ ) the roughness declined to approximately $0.3 \mathrm{~nm}$ and remained constant after 7 days of aging as shown in Figure 3c. Moreover, coherent mechanical stress-strain measurements confirmed that practically no physicomechanical changes occur in the films as a function of aging in TFA between 3 to 14 days (Table S1, Supporting
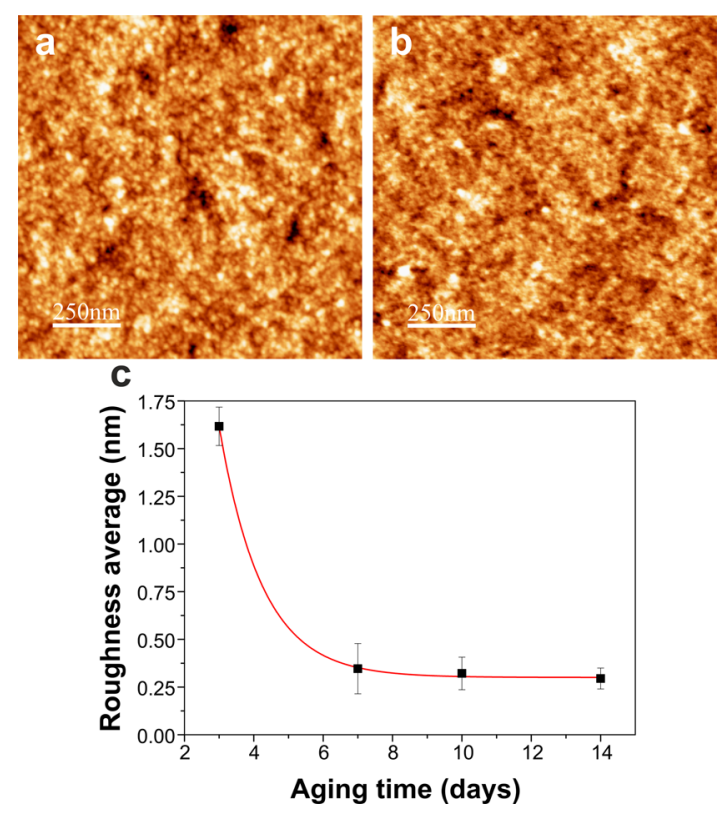

Figure 3. Noncontact mode AFM topographic images of the surfaces of pure cellulose samples cast from TFA solutions. (a) Aged in TFA for 3 days and (b) aged in TFA for 14 days. The surfaces display amorphous features with no formation of nanocrystals or cellulose nanowhiskers. (c) Results of surface roughness measurements as a function of aging days in TFA.

Information). Hence, pure cellulose bioplastics obtained from 14 days-aged TFA solutions were used as benchmarks for comparison with the ones obtained from the vegetables' wastes. We propose that TFA interacts with the microcrystalline cellulose or with the cellulose component of the wastes, breaking the hydrogen bonds between the glucose hydroxyl groups of neighboring cellulose chains (intersheet hydrogen bonds), responsible for cellulose's crystallinity. For this reason, the films generated immediately after solvent evaporation contain trifluoroacetylated cellulose. Since TFA interacts with water molecules, the substituted $\mathrm{OH}$ groups of cellulose are regenerated spontaneously under ambient conditions due to interaction of the trifluoroacetyalted moieties of cellulose with environmental humidity. We do not exclude that during cellulose aging in TFA, some glucosidic bonds, which hold together the glucose units of cellulose polymer, are also broken inducing partial cellulose depolymerization. Nevertheless, we consider this mechanism, the so-called intrasheet hydrogen bond hydrolysis, secondary for the TFA treatment times used in this work, since the physical characteristics of the obtained films remain practically unaltered in the specific times.

Chemical and Morphological Nature of the Bioplastics. Concerning the lignin component of the bioplastics, comparison of the FTIR spectra of the as-received wastes and of their derived plastics indicate existence of characteristic lignin bands in both cases as shown in Figure 4. Although the assessment of the chemical state of lignin in the bioplastics is beyond the scope of this study, it was shown that TFA can partially dissociate or reduce (break down) lignin, ${ }^{30}$ so it can be dispersed in the Bioplastic matrix in its new forms. The red arrows marked as (1) in Figure 4, parts a and b, are attributed to $\mathrm{C}=\mathrm{O}$ stretching of acetyl or carboxylic acid $\left(1740 \mathrm{~cm}^{-1}\right)$ which are also present in the structure of lignin. The blue arrows marked as (2) also in Figure 4, parts a and b, are attributed to the $\mathrm{C}=\mathrm{C}$ aromatic stretching bands (1610-1595 
a

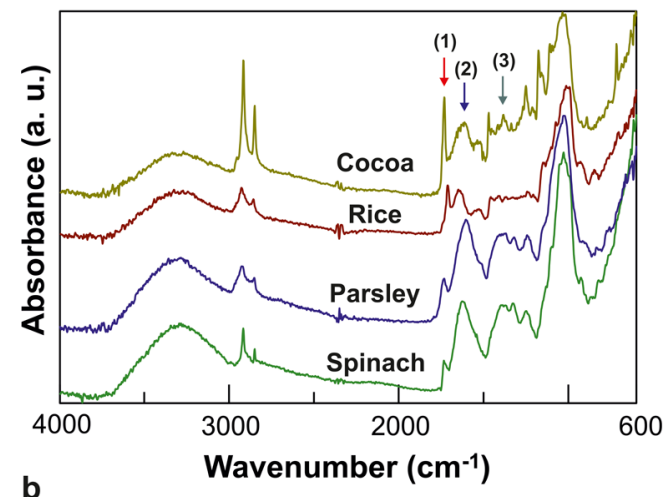

b

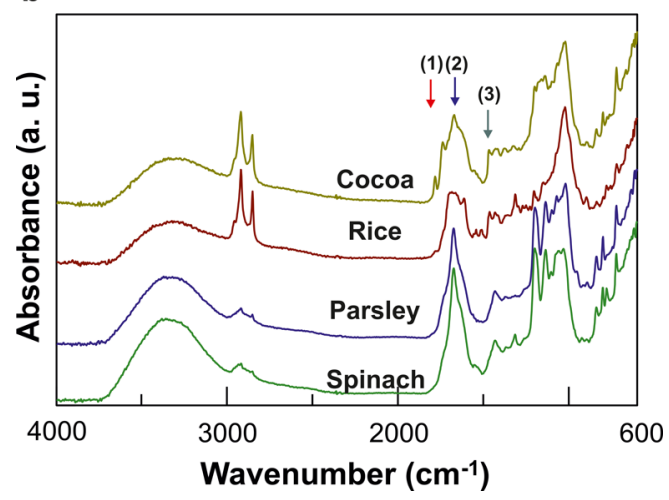

Figure 4. Comparison of FTIR spectra of (a) as-received processed vegetable wastes and (b) bioplastics after TFA digestion. The red arrows marked as 1 are attributed to $\mathrm{C}=\mathrm{O}$ stretching of acetyl or carboxylic acid $\left(1740 \mathrm{~cm}^{-1}\right)$ which are also present in the structure of lignin. The blue arrows marked as 2 are attributed to the $\mathrm{C}=\mathrm{C}$ aromatic stretching bands $\left(1610-1595 \mathrm{~cm}^{-1}\right)$ that are common to lignin. The green arrows marked as 3 can be attributed to asymmetric bending $\left(1465 \mathrm{~cm}^{-1}\right)$ in lignin $\mathrm{CH}_{3}$ bonds. The narrow bands near $1740 \mathrm{~cm}^{-1}$ in part a mostly disappear after TFA treatment as seen in part b.

$\mathrm{cm}^{-1}$ ) that are common to lignin. The green arrows marked with (3) can be attributed to asymmetric bending $\left(1465 \mathrm{~cm}^{-1}\right)$ in lignin $\mathrm{CH}_{3}$ bonds. The narrow bands near $1740 \mathrm{~cm}^{-1}$ in Figure 4 (a) mostly disappear after TFA treatment as seen in Figure 4(b). This could be due to the interaction of TFA, as TFA can hydrolyze ester linkages in lignin structure that can be correlated to partial dissociation of lignin. It should also be noted that other phytochemicals (i.e., antioxidant phenols) in these plants have $\mathrm{C}=\mathrm{C}$ aromatic bond stretching similar to lignin phenols. As shown by earlier works using anhydrous TFA, like the one used in this work, large part of lignin can remain insoluble, ${ }^{31}$ so it is highly probable that the precipitates of our procedure (Figure 1c) contain insoluble lignin. These works also revealed that hemicellulose and other noncellulosic polysaccharides are soluble in TFA during digestion. ${ }^{31}$

$\mathrm{X}$-ray diffraction spectroscopy (XRD) was used to determine the degree of crystallinity of the Bioplastic films generated from TFA solutions of the biowastes and the microcrystalline cellulose. The amorphous nature of pure cellulose bioplastics was confirmed by the existence of a very broad peak at $2 \theta=20^{\circ}$ shown in Figure 5a. The same peak is present in all the XRD spectra of the films obtained from wastes, demonstrating their amorphous nature. Moreover, these films display various crystalline peaks from $2 \theta=15^{\circ}$ to $2 \theta=46^{\circ}$. In order to identify the crystals embedded in these films, bearing in mind
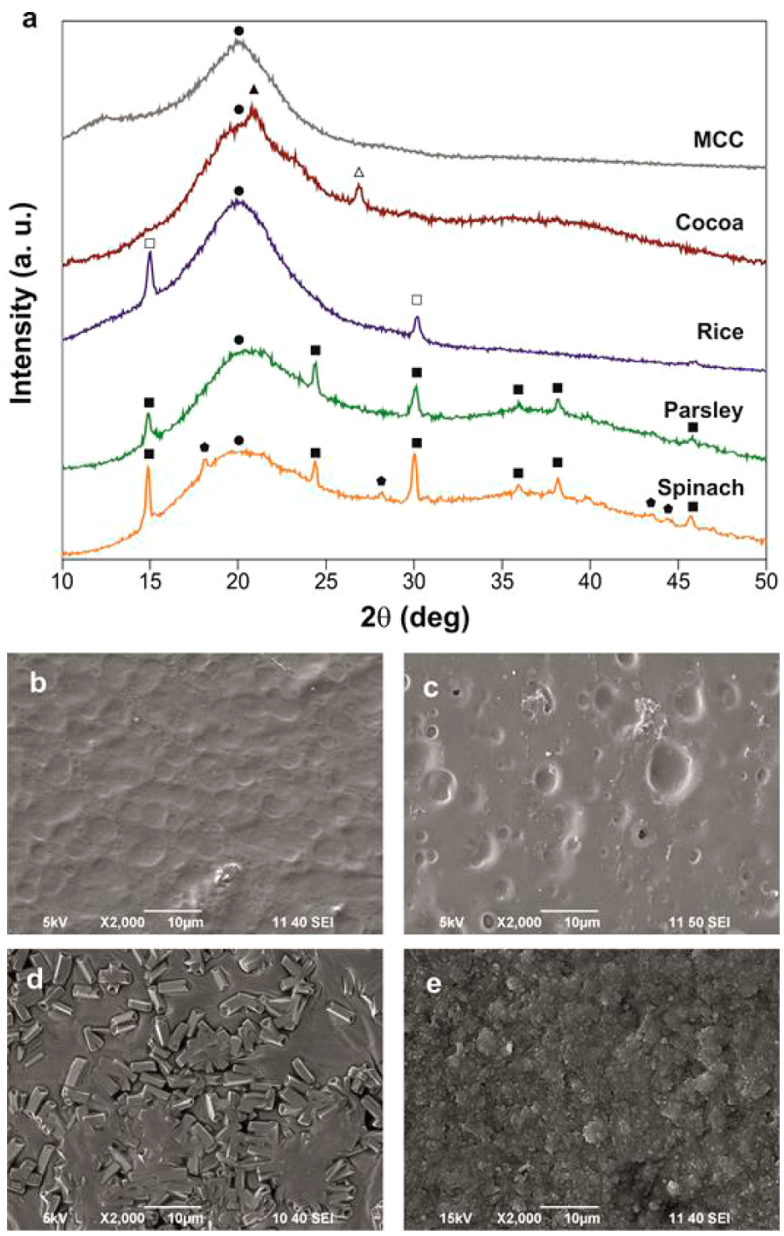

Figure 5. Morphological and structural characterization of the edible plant waste bioplastics. (a) X-ray diffractograms of pure cellulose and plant waste bioplastics. The broad band at $20^{\circ}$ is associated with the amorphous state. Other peaks are ascribed to various crystalline domains as follows: ( ) basal distance between amorphous organic chains; $(\mathbf{\Delta}) \beta V$ peak of cocoa butter in chocolate; $(\Delta) \beta V I$ peak of 1,3-distearoyl-2-oleoylglycerol in chocolate; (ם) calcium oxalate monohydrate (whewellite): (solid $\square$ ) magnesium oxalate (glushinskite). ( $\square$ ) Weddellite crystals of calcium oxalate observed in rice films. (b-e) SEM images of the surfaces of parsley, rice, spinach, and cocoa samples, respectively. In general, amorphous morphology is evident for all the films. For parsley and rice samples small islands of inorganic aggregates are detected, while for spinach film self-assembled embedded crystallites are evident. Cocoa sample shows wax-like roughness features.

that no previous XRD measurement data are available on such bioplastics, we have resorted to EDX elemental analysis measurements, presented next, as well as inductively coupled plasma spectrometry (ICP-EOS) analysis. In the case of spinach and parsley films, the XRD peaks appearing at $2 \theta=$ $15^{\circ}, 24^{\circ}, 30^{\circ}, 36^{\circ}, 38^{\circ}$, and $46^{\circ}$ are attributed to calcium oxalate monohydrate whewellite crystal structure. ${ }^{32}$ The majority of these peaks are also present in the rice films (weddellite crystals of calcium oxalate). The peaks at approximately $2 \theta=18^{\circ}, 28^{\circ}, 44^{\circ}$, and $45^{\circ}$ are attributed to magnesium oxalate glushinskite crystal structure. ${ }^{33}$ The XRD spectrum of bioplastics from cocoa pod husks highly resembles the amorphous features of cellulose films except for the presence of two peaks at $2 \theta=21^{\circ}$ and $2 \theta=27^{\circ}$. The peak at $2 \theta$ $=21^{\circ}$ is a very common peak known as $\beta \mathrm{V}$ crystalline peak of 

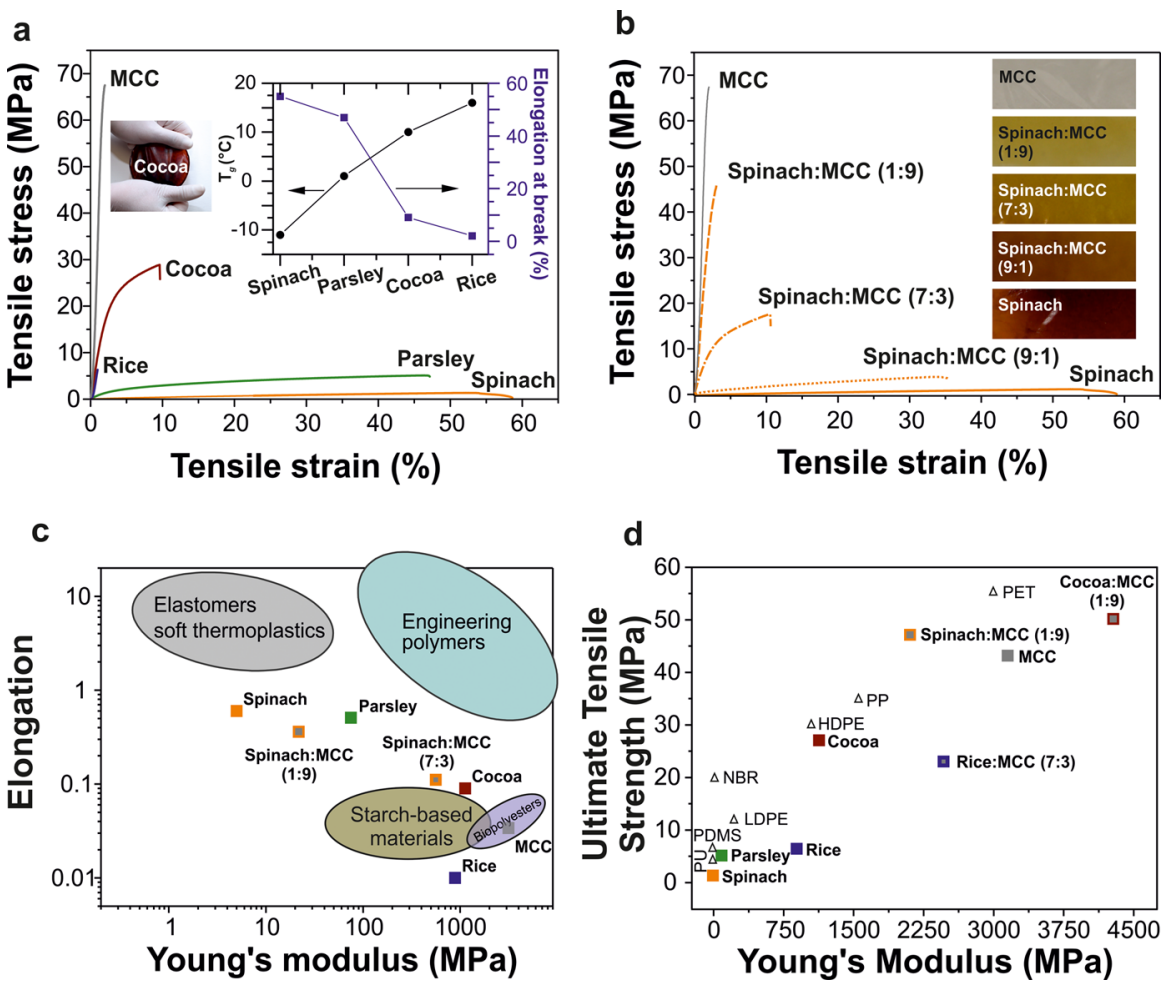

Figure 6. Mechanical properties of pure cellulose and edible plant waste bioplastics. (a) Typical stress-strain curves for pure cellulose, cocoa, rice, parsley, and spinach films. Inset shows the relationship between the glass transition temperature and the elongation at break. The photograph below shows a stretched film from cocoa pod husk waste. (b) Typical stress-strain curves for MCC:spinach blends. The physical condition of the tested samples is also shown. (c) Elongation versus Young's modulus data where the property domains of elastomers/soft thermoplastics (polyurethanes, rubbers and silicone polymers) engineering polymers, (polypropylene, polyethylene, polystyrene, polycarbonate, etc.), biopolyesters and starchbased materials (polylactic acid, poly(3-hydroxybutyrate), polycaprolactone, modified starches, etc.) are marked for comparisons. (d) Ultimate tensile strength versus Young's modulus data.

cocoa butter in chocolate. ${ }^{34}$ In general, the XRD spectrum of chocolate contains many sugar related crystal peaks ranging from $2 \theta=13^{\circ}$ to $2 \theta=27^{\circ}$, with the peak at $2 \theta=27^{\circ}$ being generally attributed to $\beta V I$ phase due to packing of $1,3-$ distearoyl-2-oleoylglycerol seeds toward $\beta$ crystallization. ${ }^{35}$ The results indicate the cocoa pod husk bioplastics are rich in cellulose and triglyceride oils. Plant-based triglycerides are very important raw materials for the synthesis of other types of biodegradable polymers. ${ }^{34}$

The micromorphology of the Bioplastic films from wastes was analyzed with scanning electron microscopy (SEM) including energy dispersive X-ray spectroscopy (EDX), where possible. Surface SEM images of bioplastics obtained from parsley (Figure $5 b$ ) indicate highly uniform amorphous surface texture with very few randomly dispersed islands of inorganic particulate aggregates. In situ EDX analysis of these regions (see Figure S2a, Supporting Information) demonstrated presence of minerals such as magnesium, potassium, sulfur, phosphorus and calcium, considered to be related to the natural biomineralization performed by plant cells and present in different shapes and sizes in plant leaves and stems. ${ }^{37-39}$ Films obtained from rice hulls also featured amorphous texture with the appearance of various particle islands somewhat similar to parsley (Figure 5c). The EDX analysis showed existence of silica, potassium and sulfur in these regions (see Figure S2b, Supporting Information). The morphology of the Bioplastic films obtained from spinach stems showed very interesting features as seen in Figure 5d. These films also showed the greatest number of crystalline peaks in the XRD spectra. Their surface is densely populated with microcrystallites embedded in the amorphous polymeric matrix. These crystals were also present in the bulk of the films. The EDX analysis of these crystallites indicated presence of calcium, magnesium, potassium, and phosphorus (see Figure S2c, Supporting Information). Finally, the overall morphology of the cocoa films appears amorphous but with unique micro features of wax-like roughness, as seen in Figure 5e. These features can be attributed to the naturally occurring triglycerides in the form of waxy fatty acids embedded in the crude fiber network of cocoa pod husks. ${ }^{34}$ EDX analysis indicated presence of elements such as magnesium, sulfur, potassium and phosphorus. However, the intensity of these elemental signals was much lower than the bioplastics obtained from spinach (see Figure S2d, Supporting Information). Cross section SEM images were also used to analyze the bulk morphological homogeneity of bioplastics. Parsley, cocoa, and spinach films had very uniform bulk structure, whereas rice films had many cracks in the bulk indicating a highly brittle and rigid Bioplastic (for representative images see Figure S2e, Supporting Information). Detailed cross-section SEM analysis on various different samples obtained from the spinach stem waste confirmed the existence of the crystals demonstrated above in Figure 5d, throughout the films but depending on the batch of waste used, different forms of self-assembled crystal structures emerged, similar to the naturally synthesized minerals on plant leaves. ${ }^{38}$ For instance, in some cases, small crystal networks forming ribbon-like features appeared (see Figure S2a, Supporting Information), and in others, formation of larger crystals randomly dispersed among the smaller crystal 
islands was observed (see Figure S2b, Supporting Information). However, the macroscopic appearances of the spinach films as well as their mechanical properties were not altered by such different forms of crystal self-assembly. Mineral inclusions are often sculpted by the plant into shapes required to support functional roles that include the storage of specific elements, the strengthening of the plant structure, the defense against pathogens and herbivores and the scattering of light for efficient photosynthesis. ${ }^{37-39}$ Such biomineral embed bioplastics synthesized by a single step can be deliberated as artificial plant leaves as they closely mimic cystolith bioceramics used for photonic engineering in plants. The microscopic mineral nodules in these bioplastics can act as light scatterers to help distribute light through the upper layers of a photonic device (i.e., solar cells) reducing losses caused by the steep gradient of illumination. ${ }^{34-36}$

Thermo-Mechanical Properties of the Bioplastics. The mechanical properties of the waste and microcrystalline cellulose-generated bioplastics and their blends are presented in Figure 6. The tensile stress-strain results show that parsley and spinach bioplastics display the highest strains of $45 \%$ and $60 \%$, respectively (Figure 6a). Cocoa films show strain of about $10 \%$, whereas microcrystalline cellulose and rice bioplastics display even less strain of approximately $3 \%$. Tensile stress at break for the amorphous cellulose films is the highest among all the films being close to $70 \mathrm{MPa}$. Films obtained from cocoa pod husks displayed approximately $30 \mathrm{MPa}$ tensile stress at break whereas rice, parsley, and spinach films displayed 7, 5, and approximately $1 \mathrm{MPa}$ stress at break values, respectively. This behavior is strongly correlated to the bio-origin and the noncellulosic constituents in the films. For instance, rice hulls are rich in silica in addition to other minerals as shown in Figure S2b of the Supporting Information. Hence, rice films cast from TFA dissolution also contain silica. This silica content can significantly alter mechanical properties of the organic films, particularly rendering them more brittle and rigid compared to parsley and spinach. In the case of cocoa pod husks, as shown earlier, a significant amount of triglycerides are present. These triglycerides are oligomeric esters (precursors for biopolymers), and are considered to be responsible for higher stresses at break and strains associated with films obtained from cocoa pod husks. Glass transition temperatures of the films were also measured, and are summarized in Table S2 of the Supporting Information. The inset in Figure 6a shows that the elongation at break is inversely correlated with the glass transition temperature of the films, a typical behavior for many amorphous conventional polymers. ${ }^{40}$

Note that plasticization of amorphous cellulose is a very challenging task. During the viscose process, cellophane is plasticized with glycerol that significantly reduces its resistance to water. With the proposed method the vegetable waste solutions can be directly blended with pure cellulose solutions to form bioplastics with tunable mechanical properties. Figure $6 \mathrm{~b}$ shows the changes in the mechanical properties of amorphous cellulose as a function of blending with spinach waste at different ratios. The inverse is also true when adding cellulose into any of the waste bioplastics; their mechanical properties can be tuned. The inset in Figure $6 \mathrm{~b}$ shows a photograph of different bioplastics obtained by blending pure microcrystalline cellulose and spinach at different ratios. Hence the use of TFA as common solvent of pure cellulose and vegetables' wastes enables a full spectrum of plasticizer action that cannot be feasible with synthetic plasticizers.
Comparisons with Engineering Polymers. Figure 6c depicts elongation versus Young's modulus results of the Bioplastic films including comparisons with common engineering polymers such as polypropylene, polyethylene, polyester, elastomers such as silicone and polyurethane elastomers, starchbased polymers, starch-polymer blends, and biopolyesters such as polycaprolactones, polylactic acids etc. As seen, waste bioplastics fill up the performance gap between synthetic polymers and conventional biopolymers. In Figure $6 \mathrm{~d}$ comparisons based on ultimate tensile strength (UTS) versus Young's modulus are presented. Amorphous pure cellulose films display high UTS at high Young's modulus comparable to poly(ethylene terephthalate) (PET), whereas parsley, spinach and rice Bioplastic films cluster close to elastomers and low density polyethylene thermoplastic. Bioplastics from cocoa pod husk can be compared with petroleum-based thermoplastics such as high density polyethylene and polypropylene. It is also possible to produce bioplastics designed to close the gap between PET and polypropylene. For instance, blend of microcrystalline cellulose with spinach (10 wt \%) would yield a property that directly falls in the middle of this gap. This proves that a wide range of mechanical properties can be designed by simply blending these vegetable waste bioplastics that many times cannot be achieved by solution or thermoform blending of conventional polymers due to incompatibility issues.

Figure 7a shows comparisons among the onset of thermal decomposition, measured by thermogravimetric analysis (TGA), of fabricated plant-waste bioplastics with various conventional plastics. Spinach bioplastics have much higher initial decomposition temperature $\left(\sim 150{ }^{\circ} \mathrm{C}\right)$ than poly(vinyl alcohol) and polyvinyl chloride, whereas amorphous cellulose has thermal degradation temperature $\left(\sim 300{ }^{\circ} \mathrm{C}\right)$ close to high performance polymers such as nylon. Note that native cellulose starts to degrade thermally at $250{ }^{\circ} \mathrm{C}$ a lower temperature ${ }^{41}$ compared to the one of the amorphous cellulose films obtained by the present method. All thermo mechanical-measurements were made on films kept under $44 \% \mathrm{RH}$ (room conditions). Parsley and cocoa bioplastics display adjacent thermal degradation points near $200{ }^{\circ} \mathrm{C}$ whereas bioplastics obtained from rice husks display even higher thermal degradation at 225 ${ }^{\circ} \mathrm{C}$, comparable to plastics such as polystyrene, polyethylene, and rubbers. The inset in Figure $7 \mathrm{a}$ displays characteristic weight loss curves of pure cellulose and parsley bioplastics measured with TGA. Pure cellulose demonstrates a single welldefined thermal degradation peak whereas waste-based bioplastics such as parsley have multiple thermal degradation peaks. This can be attributed to the existence of organic multicomponents in biowaste samples.

Interaction with Water. Finally, another critical property of polymers, the water adsorption, is presented in Figure $7 \mathrm{~b}$ for all the bioplastics developed with our method as well as for cellulose acetate and viscose (cellophane) ${ }^{42}$ for comparison reasons. At low to medium humidity levels $(<50 \%)$, all generated bioplastics exhibit lower water adsorption than viscose, and in particular, cocoa and rice bioplastics demonstrate the lowest values comparable to cellulose acetate. Cocoa, rice, and pure cellulose bioplastics maintain a relatively low adsorption behavior also at $80 \% \mathrm{RH}$, whereas parsley and spinach demonstrate higher water adsorption, comparable to viscose. At $100 \% \mathrm{RH}$, cocoa, spinach, and parsley bioplastics absorb an amount of water close to $40 \%$ of their weight just as cellophane, whereas rice and cellulose demonstrate water uptake close to $25 \%$. The inset in Figure $7 \mathrm{~d}$ shows photographs 
a

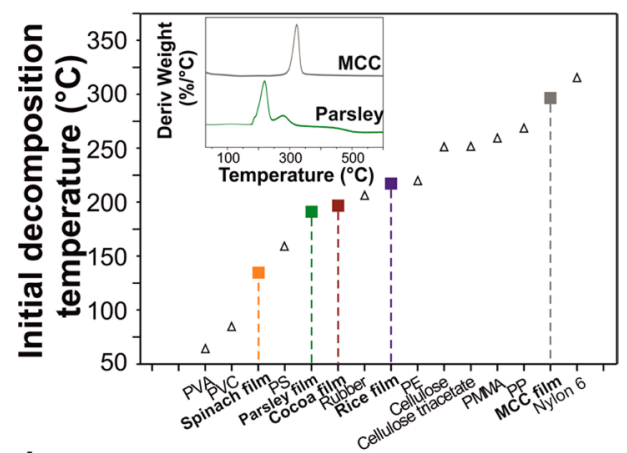

b

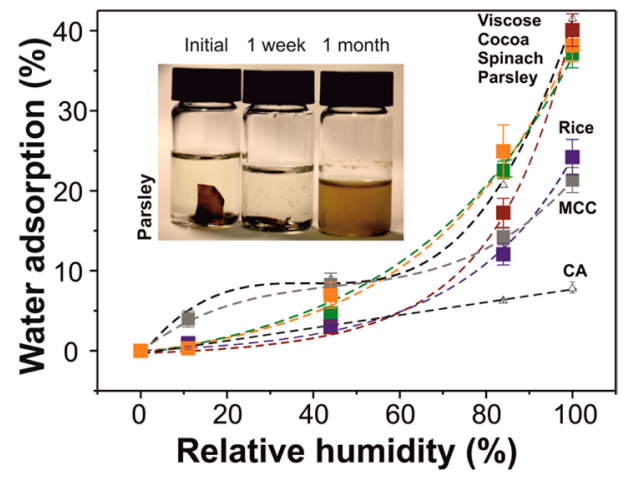

Figure 7. Thermal decomposition and water adsorption isotherms. (a) Thermal decomposition temperatures of the bioplastics in comparison to various other conventional plastics such as poly(vinyl alcohol) (PVA), polypropylene (PP), polyethylene (PE), poly(vinyl chloride) (PVC), polystyrene (PS), poly(methyl methacrylate) (PMMA), and nylon. The initial decomposition temperature (minimum thermal decomposition temperature) indicates the onset of polymer chain scission and loss of structural stability. The inset shows thermal weight loss profiles of cellulose and parsley bioplastics. (b) Water adsorption isotherms for vegetable and cellulose bioplastics. Viscose and cellulose acetate (CA) controls are also shown. The inset shows the degradation of parsley bioplastic in water with time. Data are expressed as mean \pm s.d. $(n \geq 10)$.

of degradation of parsley Bioplastic soaked in deionized water. The environmental degradation of the generated bioplastics is implied by the fact that they slowly disintegrate in water as shown in these photos. Within 1 week, the films get swollen and start to fragment into smaller pieces and fibers, whereas at the end of one month they completely disintegrate in water changing its color. Similar behaviors were monitored for all the developed biofilms based on vegetable and cereal wastes but with different time dynamics.

\section{CONCLUSIONS}

In this work, we demonstrated for the first time that industrially processed wastes from edible cereals and vegetables rich in cellulose can be transformed into bioplastics by simply aging them in trifluoroacetic acid (TFA) solutions regardless of their bio-origin. Depending on the plant species, biopolymers were found to display diverse mechanical properties ranging from brittle and rigid to soft and stretchable. Blending these vegetable waste solutions with TFA solutions of pure cellulose, all-natural plasticization of amorphous cellulose is achieved. These new bioplastics can replace many nondegrading plastics, preserving the environment, and open up new avenues toward tailoring mechanical properties of cellulose for applications in packaging and biomedicine as well as designing new feedstock for biofuel synthesis.

\section{ASSOCIATED CONTENT}

\section{S Supporting Information}

Mechanical properties, glass transition temperatures, photo of fibrillar film from non-dissolvable portion of plant waste solutions, analysis of surface composition and morphological characterization, and SEM images of bioplastic surfaces. This material is available free of charge via the Internet at http:// pubs.acs.org.

\section{AUTHOR INFORMATION}

\section{Corresponding Authors}

*(I.S.B.) E-mail: ilker.bayer@iit.it.

*(A.A.) E-mail: athanassia.athanassiou@iit.it.

\section{Author Contributions}

S.G.: Fabrication and characterization of the bioplastics. J.A.HG., L.C., and F.P: Spectroscopic, mechanical and thermal characterization of the bioplastics. R.R.: Electron microscopy characterization of the bioplastics. R.C.: Interpretation and project leading. I.S.B.: Concept generation, preliminary experiments and manuscript writing. A.A.: Project development and leading, manuscript writing and interpretation. All authors have been involved in the redaction of the manuscript.

\section{Notes}

The authors declare no competing financial interests.

\section{ACKNOWLEDGMENTS}

The authors acknowledge the European Association of Fruit and Vegetable Processors (PROFEL) for providing logistical and materials support and consulting.

\section{REFERENCES}

(1) Yamamoto, T.; Yasuhara, A. Quantities of bisphenol A leached from plastic waste samples. Chemosphere 1999, 38, 2569-2576.

(2) Lithner, D.; Larsson, A.; Dave, G. Environmental and health hazard ranking and assessment of plastic polymers based on chemical composition. Sci. Total Environ. 2011, 409, 3309-3324.

(3) Teuten, E. L.; et al. Transport and release of chemicals from plastics to the environment and to wildlife. Philos. Trans. R. Soc. London, B 2009, 364, 2027-2045.

(4) Tremblay, J.-F. Tackling the plastic waste problem. Chem. Eng. News 2013, 91, 26.

(5) Rochman, C. M.; Hoh, E.; Hentschel, B. T.; Kaye, S. Long-Term Field Measurement of Sorption of Organic Contaminants to Five Types of Plastic Pellets: Implications for Plastic Marine Debris. Environ. Sci. Technol. 2013, 47, 1646-1654.

(6) Eriksson, O.; Finnveden, G. Plastic waste as a fuel - CO2-neutral or not? Energy Environ. Sci. 2009, 2, 907-914.

(7) Law, K. L.; et al. Plastic Accumulation in the North Atlantic Subtropical Gyre. Science 2010, 329, 1185-1188.

(8) Barnes, D. K. A.; Galgani, F.; Thompson, R. C.; Barlaz, M. Accumulation and fragmentation of plastic debris in global environments. Philos. Trans. R. Soc., London B 2009, 364, 1985-1998.

(9) Morritt, D.; Stefanoudis, P. V.; Pearce, D.; Crimmen, O. A.; Clark, P. F. Plastic in the Thames: a river runs through it. Mar. Pollut. Bull. 2014, 78, 196-200.

(10) Garcia, H.; Rui Ferreira, R.; Celso Martins, C.; Andreia, F.; Sousa, A. F.; Carmen, S. R.; Freire, C. S. R; Silvestre, A. J. D.; Kunz, W.; Rebelo, L. P. N.; Pereira, C. S. Ex Situ Reconstitution of the Plant Biopolyester Suberin as a Film. Biomacromolecules 2014, DOI: $10.1021 / \mathrm{bm} 500201 \mathrm{~s}$. 
(11) AWARENET. Handbook for the prevention and minimization of waste and valorisation of by-products in European agro-food Industries; Gaiker Foundation: Bilbao, Spain, 2004.

(12) Singh, A.; Kuila, A.; Adak, S.; Bishai, M.; Barnejee, R. Utilization of vegetable wastes for bioenergy generation. Agr. Res. 2012, 1, 213222.

(13) Sathitsuksanoh, N.; Xu, B.; Zhao, B. Y.; Zhang, Y. H. P. Overcoming Biomass Recalcitrance by Combining Genetically Modified Switchgrass and Cellulose Solvent-Based Lignocellulose Pretreatment. PLoS One 2013, 8, e73523.

(14) Pingali, S. V.; Urban, V. S.; Heller, W. T.; McGaughey, J.; O’Neill, H.; Foston, M.; Myles, D. A.; Ragauskas, A.; Evans, B. R. Breakdown of Cell Wall Nanostructure in Dilute Acid Pretreated Biomass. Biomacromolecules 2010, 11, 2329-2335.

(15) Onda, A.; Ochi, T.; Yanagisawa, K. Selective hydrolysis of cellulose into glucose over solid acid catalysts. Green Chem. 2008, 10, 1033-1037.

(16) Nishiyama, Y.; Langan, P.; Chanzy, H. Crystal structure and hydrogen-bonding system in cellulose 1 beta from synchrotron X-ray and neutron fiber diffraction. J. Am. Chem. Soc. 2002, 124, 9074-9082.

(17) Zhao, H. B.; Holladay, J. E.; Kwak, J. H.; Zhang, Z. C. Inverse temperature-dependent pathway of cellulose decrystallization in trifluoroacetic acid. J. Phys. Chem. B 2007, 111, 5295-5300.

(18) Kraemer, E. O. Molecular weights of celluloses. Ind. Eng. Chem. 1938, 30, 1200-1203.

(19) Gilbert, R. D. Cellulosic liquid crystals: Overview. In Polymeric materials encyclopedia; CRC: Boca Raton, FL, 1996; Vol. 2C.

(20) Scott, B. F.; et al. Trifluoroacetate profiles in the Arctic, Atlantic, and Pacific Oceans. Environ. Sci. Technol. 2005, 39, 6555-6560.

(21) Kim, B. R.; Suidan, M. T.; Wallington, T. J.; Du, X. Biodegradability of trifluoroacetic acid. Environ. Eng. Sci. 2000, 17, 337-342.

(22) Katz, J. J. Anhydrous trifluoroacetic acid as a solvent for proteins. Nature 1954, 174, 509.

(23) Pfaltzgraff, L. A.; De Bruyn, M.; Cooper, E. C.; Budarin, V.; Clark, J. H. Food waste biomass: a resource for high-value chemicals. Green Chem. 2013, 15, 307-314.

(24) Sanderson, K. A Chewy problem. Nature 2011, 474, S12-S14.

(25) Ouzounidou, G.; Papadopoulou, K. K.; Asfi, M.; Mirtziou, I.; Gaitis, F. Efficacy of different chemicals on shelf life extension of parsley stored at two temperatures. Int. J. Food Sci. Technol. 2013, 48, $1610-1617$.

(26) Pinkert, A.; Goeke, D. F.; Marsh, K. N.; Pang, S. S. Extracting wood lignin without dissolving or degrading cellulose: investigations on the use of food additive-derived ionic liquids. Green Chem. 2011, 13, 3124-3136.

(27) Cavalcanti, R. N.et al. Natural product extraction: principles and applications; RSC Publisher: London, 2013.

(28) Hertrampf, J. W., Piedad-Pascual, F. Handbook on ingredients for aquaculture feeds; Springer Netherlands: Dordrecht, The Netherlands, 2000.

(29) Ritcey, A. M.; Holme, K. R.; Gray, D. G. Cholesteric properties of cellulose-acetate and triacetate in trifluoroacetic acid. Macromolecules 1988, 21, 2914-2917.

(30) Dong, D. X.; et al. Using trifluoroacetic acid to pretreat lignocellulosic biomass. Biomass Bioenerg. 2009, 33, 1719-1723.

(31) Morrison, I. M.; Stewart, D. Plant cell wall fragments released on solubilisation in trifluoroacetic acid. Phytochemistry 1998, 49, $1555-1563$.

(32) East, C. P.; Fellows, C. M.; Doherty, W. O. S. Modeling the coprecipitation of silica and calcium oxalate in sugar solutions. J. Food Eng. 2014, 121, 166-173.

(33) Kolo, K.; Claeys, P. In vitro formation of Ca-oxalates and the mineral glushinskite by fungal interaction with carbonate substrates and seawater. Biogeosciences 2005, 2, 277-293.

(34) Le Reverend, B. J. D.; Fryer, P. J.; Bakalis, S. Modelling crystallization and melting kinetics of cocoa butter in chocolate and application to confectionery manufacturing. Soft Matter 2009, 5, 891902.
(35) Schenk, H.; Peschar, R. Understanding the structure of chocolate. Radiat. Phys. Chem. 2004, 71, 829-835.

(36) Guner, F. S.; Yagci, Y.; Erciyes, A. T. Polymers from triglyceride oils. Prog. Polym. Sci. 2006, 31, 633-670.

(37) Gal, A.; Brumfeld, V.; Weiner, S.; Addadi, L.; Oron, D. Certain Biominerals in Leaves Function as Light Scatterers. Adv. Mater. 2012, 24, OP77-OP83.

(38) Ball, P. Why leaves have stones. Nat. Mater. 2012, 11, 271-271.

(39) Webb, M. A. Cell-mediated crystallization of calcium oxalate in plants. Plant Cell 1999, 11, 751-761.

(40) Roig, F.; Dantras, E.; Dandurand, J.; Lacabanne, C. Influence of hydrogen bonds on glass transition and dielectric relaxations of cellulose. J. Phys. D Appl. Phys. 2011, 44, 045403.

(41) Kholodovych, V.; Welsh, W. J. Thermal-Oxidative Stability and Degradation of Polymers, Physical Properties of Polymers Handbook; Mark, J. E., Ed.; Springer: New York, 2007; pp 927-938.

(42) Kohler, R.; Alex, R.; Brielmann, R.; Ausperger, B. A new kinetic model for water sorption isotherms of cellulosic materials. Macromol. Symp. 2006, 244, 89-96. 